\title{
Antracnose em Noni (Morinda citrifolia L.) no Estado de Goiás
}

\author{
Anthracnose in Noni (Morinda citrifolia L.) in the State of Goiás \\ Antracnosis en Noni (Morinda citrifolia L.) en el Estado de Goiás
}

\author{
Mônica Lau da Silva Marques \\ ORCID: https://orcid.org/0000-0002-2794-0815 \\ Instituto Federal de Educação, Ciência e Tecnologia Goiano, Brasil \\ E-mail: monica.lau@ifgoiano.edu.br \\ Jéssica Maria Israel de Jesus \\ ORCID: https://orcid.org/0000-0002-8261-9936 \\ Universidade de São Paulo, Brasil \\ E-mail: jessicamariaisrael@gmail.com \\ Raquel Silva Firmiano \\ ORCID: https://orcid.org/0000-0002-9079-200X \\ Instituto Federal de Educação, Ciência e Tecnologia Goiano, Brasil. \\ E-mail: raquels.firmiano@gmail.com \\ Adriana Lau da Silva Martins \\ ORCID: https://orcid.org/0000-0002-0851-5522 \\ Centro Universitário Geraldo Di Biase, Brasil \\ E-mail: adralmartins@ hotmail.com.br \\ Estelita Marques \\ https://orcid.org/0000-0001-7712-9687 \\ Secretaria de Estado de Educação do Estado do Mato Grosso, Brasil \\ E-mail: estelita_marques31@hotmail.com \\ Luís Sérgio Rodrigues Vale \\ ORCID: https://orcid.org/0000-0002-6303-9063 \\ Instituto Federal de Educação, Ciência e Tecnologia Goiano, Brasil. \\ E-mail: luis.sergio@ifgoiano.edu.br \\ Paula Gonçalves Silva \\ ORCID: https://orcid.org/0000-0003-1638-3416 \\ Instituto Federal de Educação, Ciência e Tecnologia Goiano, Brasil \\ E-mail:paulasilvag @outlook.com \\ Raissy Arielly Marques da Silva Raizel \\ ORCID: https://orcid.org/0000-0002-4813-3182 \\ Universidade Federal Rural do Rio de Janeiro, Brasil \\ E-mail: raissymarques7@gmail.com \\ Valter dos Santos Marques \\ ORCID: https://orcid.org/0000-0002-9690-2361 \\ Instituto Federal de Educação, Ciência e Tecnologia Goiano, Brasil \\ E-mail: mltopografiavalter@gmail.com \\ Aurélio Ludovico de Almeida Martinez \\ ORCID: https://orcid.org/0000-0001-8095-7088 \\ Instituto Federal de Educação, Ciência e Tecnologia Goiano, Brasil \\ E-mail: aurélio.martinez@ifgoiano.edu.br
}

\begin{abstract}
Resumo
O Noni (Morinda citrifolia L.) é uma planta nativa do Sudeste da Ásia que produz frutos com importantes propriedades nutricionais e terapêuticas. Por isso nos últimos anos o cultivo tem se expandido em todo o Brasil. Entretanto com o aumento da área cultivada, a ocorrência de fitopatologias tornou-se mais frequente, o que pode comprometer a produtividade da cultura. Diante disso, objetivou-se relatar a ocorrência de antracnose em plantas de noni no Estado de Goiás. Foram realizados isolamentos a partir de lesões foliares de plantas sintomáticas coletadas nos municípios de Ceres e Goianésia. O teste de patogenicidade dos isolados foi realizado. Para a caracterização morfológica do patógeno, foram confeccionadas lâminas e as estruturas foram observadas em microscopia de luz. Os sintomas iniciais nas folhas inoculadas eram pequenas manchas circulares de coloração marrom-clara, circulares e irregulares que gradualmente aumentaram, tornando-se escuras e com pontuações alaranjadas. As colônias fúngicas apresentaram rápido crescimento e esporulação intensa, coloração acinzentada com margens brancas. Micélio hialino septado e ramificado. Conidióforos simples, alongados. Os conídios hialinos, unicelulares, retos, cilíndricos ou elípticos. Com base nas características morfológicas pode-se concluir que o fungo do gênero Colletotrichum é o agente causal da antracnose no noni no Estado de Goiás.
\end{abstract}

Palavras-chave: Colletotrichum sp.; Caracterização; Fruticultura; Planta medicinal. 


\begin{abstract}
Noni (Morinda citrifolia L.) is a plant native to Southeast Asia that produces fruits with important nutritional and therapeutic properties. Therefore, in recent years, cultivation has expanded throughout Brazil. However, with the increase in the cultivated area, the occurrence of phytopathologies has become more frequent, which can compromise the productivity of the crop. Therefore, the objective was to report the occurrence of anthracnose in noni plants in the State of Goiás. Isolations were performed from leaf lesions of symptomatic plants collected in the municipalities of Ceres and Goianésia. The pathogenicity test of the isolates was performed. For the morphological characterization of the pathogen, slides were made and the structures were observed under light microscopy. The initial symptoms in the inoculated leaves were small circular spots of light brown color, circular and irregular, which gradually increased, becoming dark and with orange punctuations. The fungal colonies showed rapid growth and intense sporulation, grayish color with white margins. Hyaline mycelium septate and branched. Simple, elongated conidiophores. Hyaline, single-celled, straight, cylindrical or elliptical conidia. Based on the morphological characteristics, it can be concluded that the fungus of the genus Colletotrichum is the causative agent of anthracnose in noni in the State of Goiás.
\end{abstract}

Keywords: Colletotrichum sp.; Description; Fruit growing; Medicinal plant.

\title{
Resumen
}

Noni (Morinda citrifolia L.) es una planta originaria del sudeste asiático que produce frutos con importantes propiedades nutricionales y terapéuticas. Por lo tanto, en los últimos años, el cultivo se ha expandido por todo Brasil. Sin embargo, con el aumento de la superficie cultivada, se ha hecho más frecuente la aparición de fitopatologías, que pueden comprometer la productividad del cultivo. Por tanto, el objetivo fue reportar la ocurrencia de antracnosis en plantas de noni del estado de Goiás, se realizaron aislamientos de lesiones foliares de plantas sintomáticas recolectadas en los municipios de Ceres y Goianésia. Se realizó la prueba de patogenicidad de los aislados. Para la caracterización morfológica del patógeno se realizaron portaobjetos y se observaron las estructuras al microscopio óptico. Los síntomas iniciales en las hojas inoculadas fueron pequeñas manchas circulares de color marrón claro, circulares e irregulares, que aumentaron gradualmente, oscureciendo y con puntuacion anaranjada. Las colonias de hongos mostraron crecimiento rápido e intensa esporulación, color grisáceo con márgenes blancos. Micelio hialino septado y ramificado. Conidióforos alargados y simples. Conidios hialinos, unicelulares, rectos, cilíndricos o elípticos. Con base en las características morfológicas, se puede concluir que el hongo del género Colletotrichum es el agente causante de la antracnosis en el noni en el Estado de Goiás.

Palabras clave: Colletotrichum sp.; Descripción; Fruticultura; Planta medicinal.

\section{Introdução}

O noni (Morinda citrifolia L.) é uma planta pertencente à família Rubiaceae originária do Sudeste Asiático e Austrália. Cultivada em regiões tropicais, apresenta porte pequeno, 3 à 10m de altura quando adulta. Conhecida vulgarmente como: noni, índia mulberry, iada, nono, canary, Wood ou mengkudu, seu cultivo e uso vem crescendo rapidamente no Brasil (Nelson, 2005; Wang, Su \& Chen 2001). Está presente em várias partes do mundo como as regiões tropicais da África (Centro e Sul), Caribe, Austrália, China, Malásia, Indonésia e Índia. É uma espécie que se adapta muito bem às regiões costeiras, desde o nível do mar até $400 \mathrm{~m}$ de altitude, é tolerante a solos salinos e condições de seca (Sousa, et al., 2010; Lübeck \& Hannes, 2001).

Conhecido como uma planta medicinal, o noni é utilizado há muito tempo pelos polinésios, para o tratamento de diversas doenças, como: diabetes, câncer, hipertensão, desordens menstruais, artrite e, ainda, como antimicrobiano, antiinflamatório, antioxidante, dentre outros (Rao \& Subramanian, 2009; West, et al., 2007; Potterat \& Hamburger, 2007; León \& Poveda, 2000). Atualmente, os pesquisadores vêm demonstrando um interesse crescente por esta planta e suas propriedades. Mais de cem metabólitos já foram identificados, dentre os quais os compostos fenólicos, ácidos orgânicos e alcaloides (Correia, et al., 2011). Aproximadamente 200 moléculas bioativas podem ser derivadas de noni, os efeitos desses compostos estão relacionados à atividade antibacteriana, antioxidante, antiviral, antifúngica, antitumoral, anti-helmíntica, analgésica, antiinflamatória, hipotensora e imunoestimulante, entretanto, os aspectos que regem o comportamento biológico dessas moléculas, isoladamente ou em combinação, não estão totalmente definidos (De Sousa, et al., 2017; Singh, 2012; Wang, et al., 2002).

O cultivo comercial de noni é uma atividade relativamente recente no Brasil, que tem sido impulsionado devido às propriedades medicinais da planta. Porém, á medida que a cultura começou a ser cultivada comercialmente tornou-se mais 
exposta apresentando suscetibilidade ao ataque de diversos patógenos como Cephaleuros minimus, Colletotrichum sp., Guignardia morindae, Phellinus noxius, Phytopthora sp., Rhizopus sp. e Sclerotium rolfisii (Rethinam \& Sivaraman 2007), Alternaria alternata (Hubballi, et al., 2010) e Myrothecium roridum (Poltronieri, et al., 2009).

De acordo com Carnaúba, et al., (2007) e Bonaldo, et al., (2011), para as regiões tropicais e subtropicais o gênero Colletotrichum é um dos mais importantes fungos fitopatogênicos. O gênero causa antracnose em diversas culturas, sendo esta uma doença economicamente importante. Diante disso, objetivou-se a relatar a ocorrência de antracnose em plantas de Noni no interior do Estado de Goiás, Brasil.

\section{Metodologia}

Folhas de Noni apresentando machas marrons circulares (Figura 1) foram coletadas de plantas localizadas nos municípios de Ceres e Goianésia, no Estado de Goiás e encaminhadas ao laboratório de Microbiologia do Departamento de Ciências Agrárias do Instituto Federal Goiano - Campus Ceres- GO. O isolamento indireto do patógeno foi realizado. Para tanto, os fragmentos de folhas com lesões foram desinfestados em uma sequência de solução de álcool 70\% por 30 segundos, hipoclorito de sódio $2 \%$ por 2 minutos e posteriormente enxagues três vezes em água destilada esterilizada. Com o auxílio de uma pinça flambada, os fragmentos foram transferidos para placas de Petri com meio de cultura ágar-ágar. As placas foram incubadas por 48 horas a $25^{\circ} \mathrm{C}$, em fotoperíodo de $12 \mathrm{~h} / \mathrm{luz}$, até o crescimento do micro-organismo. Após este período, o micélio da colônia obtida foi transferido para placas de Petri contendo meio de cultura, batata, dextrose e ágar (BDA) para a multiplicação do patógeno. Posteriormente foram produzidas culturas monospóricas (acondicionadas em geladeira a $5^{\circ} \mathrm{C}$ ).

Figura 1. A-B - Folhas sintomáticas coletadas.

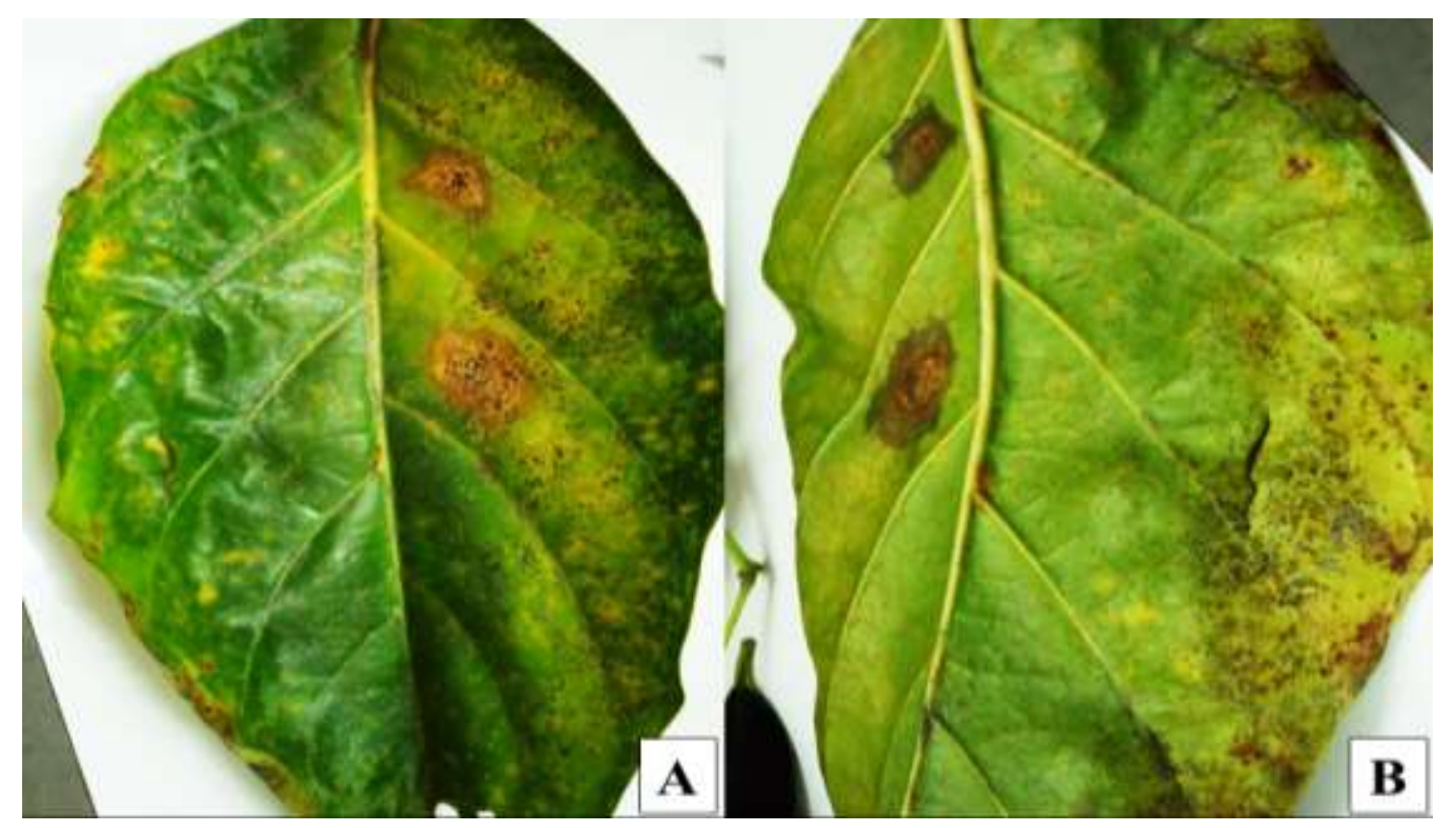

Fonte: Autores.

Para a identificação morfológica do isolado, foram observadas as características das colônias e estruturas reprodutivas. Para tanto, após o crescimento das culturas em BDA, as estruturas foram observadas em microscopia de luz (Microscópio Olympus Cover - Bx41 com auxílio do softawere Dinocapture 2.0) sob o aumento de 40x. A coloração dos conídios também foi observada, e as características foram comparadas com as descritas para o gênero na literatura (Barnett \& Hunter, 1998, Mordue, 1971). 
O teste de patogenicidade foi realizado para confirmar os postulados de Koch (1881). Para tanto a inoculação foi feita com discos de micélio $(5 \mathrm{~mm})$ retirados das bordas da colônia e depositados sobre folhas sadias, já as testemunhas foram inoculadas com discos contendo apenas BDA, as placas foram incubadas a $25{ }^{\circ} \mathrm{C}$, em fotoperíodo de $12 \mathrm{~h} / \mathrm{luz}$. O teste foi realizado com cinco repetições e executado duas vezes, com avaliação diária dos sintomas. Após cinco dias de incubação o reisolamento foi realizado para confirmar a correspondência com a identidade do organismo inoculado, validando o teste.

\section{Resultados e Discussão}

As colônias fúngicas apresentaram rápido crescimento e esporulação intensa. A coloração acinzentada, margens brancas, com formação de massas alaranjadas contendo as estruturas reprodutivas (Figura 2-A). Micélio hialino septado e ramificado. Acérvulo arredondado, ceroso, subepidérmico, tipicamente escuro, com presença de setas escuras na borda ou entre os conidióforos (Figura 2-B). Conidióforos simples, alongados. Os conídios hialinos, unicelulares, retos, cilíndricos ou elípticos medindo 8,73-15,59 × 3,47-5,32 $\mu \mathrm{m}$ (Figura 1 - E e F). As características encontradas assemelham-se as descritas na literatura para o gênero Colletotrichum (Barnett \& Hunter, 1998, Mordue, 1971). De acordo com Sutton (1992), em meio de cultura, fungos do gênero Colletotrichum formam colônias variáveis, de coloração branco-gelo a cinza escuro, com quantidade variável de micélio aéreo. Sendo variável também a produção de estruturas reprodutivas.

Figura 2. A - Colônia de Colletotrichum sp. B - Setas e conídios de Colletotrichum sp. observados em microscopia de luz sob (Microscópio Olympus Cover - Bx41, aumento de 40x). C-D - Conídios (Microscópio Olympus Cover - Bx41, aumento de 40x).

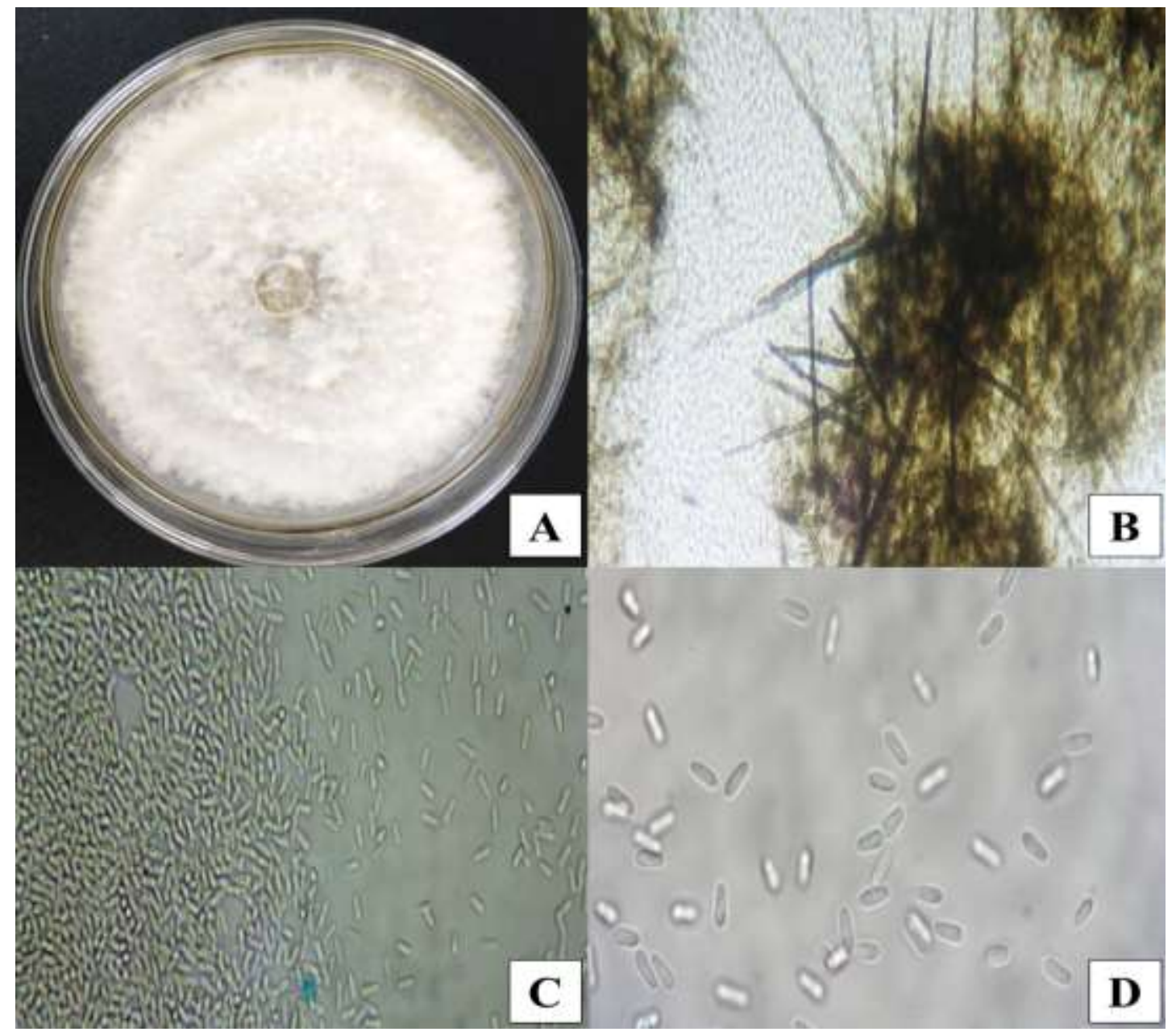

Fonte: Autores. 
Por meio do teste de patogenicidade houve a reprodução dos sintomas (lesões foliares), o re-isolamento confirmou a correspondência entre o organismo inoculado e o reisolado. Os sintomas iniciais nas folhas inoculadas eram pequenas manchas circulares de coloração marrom-clara, circulares e irregulares que gradualmente aumentaram e tornando-se escuras e com pontuações alaranjadas. Sintomas semelhantes observados por Silva, et al. (2019) sete dias após inocular folhas de noni com Colletotrichum siamense e por Ayvar- Serna, et al. (2018) aos oito dias após a inoculação com C. tropicale.

Em bioensaio de patogenicidade em frutos de noni inoculados com isolados de Colletotrichum sp. realizado por Bonaldo, et al., (2011), os autores observaram o surgimento de lesões necróticas nos frutos das quais posteriormente foi possível isolar o fungo novamente. Sintomas semelhantes aos relatados por Hubballi, et al. (2012), Kumar, et al. (2012) e Ayvar-Serna, et al. (2018) ao inocularem frutos de M. citrifolia com C. gloeosporioides, Colletotrichum spp. e Colletotrichum tropicale respectivamente, onde os frutos apresentaram a formação de lesões necróticas típicas de antracnose.

No Brasil o gênero Colletotrichum já foi associado à ocorrência de antracnose em noni na cidade de Sinop no Mato Grosso (Bonaldo, et al., 2011) e no Amazonas (Silva, et al., 2019). Ocorrendo também relatos em outros países como o México (Ayvar-Serna, et al., 2018) e a Índia (Hubballi, et al., 2010; Kumar, et al., 2012). De acordo com Sousa et al. (2010), as plantas adultas e as mudas de noni resistem ao ataque de insetos ou fitopatógenos. Em viveiros de produção de mudas, no caso de ocorrência de doenças, a primeira atitude recomendada é a diminuição da irrigação e a remoção do sombreamento. Além disso, a eliminação imediata das plântulas doentes é outra medida a ser tomada.

Com base nas características morfológicas da colônia (coloração, formato e formação de massas alaranjadas) e dos conídios (cor, formato, presença de setas e etc), o isolado foi identificado como pertencente ao gênero Colletotrichum, sendo este o primeiro relato de ocorrência deste fungo causando antracnose em folhas de Morinda citrifolia L. no interior do Estado de Goiás, Brasil.

\section{Conclusão}

O fungo do gênero Colletotrichum é o agente causal da antracnose em folhas de Noni nos municípios de Ceres e Goianésia no Estado de Goiás.

\section{Referências}

Ayvar-Serna, S., Díaz-Nájera, J. F., Vargas-Hernández, M., Plancarte-Galán, P. J., Alvarado-Gómez, O. G., Tejeda-Reyes, M. A., \& Mena-Bahena, A. (2018). Colletotrichum tropicale causal agent of anthracnose on noni plants (Morinda citrifolia) in Guerrero, Mexico. Plant Pathology \& Quarantine, 8(2), p.165-169.

Barnett, H. L. \& Hunter, B. B. (1998). Illustrated Genera of Imperfect Fungi, St. Paul, APS Press.

Bonaldo, S. M., Santos, B. T., Rondon, M. N., \& Trento, R. A. (2011). Ocorrência de Antracnose em Morinda citrifolia L. (Rubiales: Rubiaceae) em SINOP/MT. Revista de Ciências Agro-Ambientais, Alta Floresta-MT, 9 (2), 301 - 305.

Carnaúba, J. P., Sobral, M. F., Amorim, E. P. Da R., Silva, A. P., \& Silva, K. M. M. (2007). Ocorrência de Colletotrichum gloeosporioides em Mimosa caesalpinaefolia Benth no estado de Alagoas. Summa Phytopathologica, 33 (3), 313.

Correia, A. A. S., Gonzaga, M. L. C., Aquino, A. C., Souza, P. H. M., Figueiredo, R. W., \& Maia, G. A. (2011). Caracterização química e físico-química da polpa do noni (Morinda citrifolia) cultivado no estado do Ceará. Alimentos e Nutrição, 22, 609-615.

De Sousa, B. C., Miguel, C. B., Rodrigues, W. F., Machado, J. R., Da Silva, M. V., Da Costa, T. A., Lazo-Chica, J. E., Degasperi, T. P., Sales-Campos, H., Bucek, E. U., \& Oliveira, C. J. F. (2017). Effects of short-term consumption of Morinda citrifolia (Noni) fruit juice on mice intestine, liver and kidney immune modulation. Food and Agricultural Immunology, 28(3), 528-542. https://doi.org/10.1080/09540105.2017.1306492

Hubballi, M., Nakkeeran, S., \& Raguchander, T. (2012). First report of anthracnose on noni caused by Colletotrichum gloeosporioides in India. Archives of phytopathology and plant protection, 45 (3), 276-279.

Hubballi, M., Nakkeeran, S.; Raguchander, T.; Anand, T.; \& Samiyappan, R. (2010). Effect of environmental conditions on growth of Alternaria alternata causing leaf blight of noni. World Journal of Agricultural Sciences, 6 (2), 171-177.

Koch, R. (1881). Methods for the Study of Pathogenic Organisms. Mitteilungen aus dem Kaiserlichen Gesundheitsamte 2,1-48. 
Research, Society and Development, v. 10, n. 2, e32310212671, 2021

(CC BY 4.0) | ISSN 2525-3409 | DOI: http://dx.doi.org/10.33448/rsd-v10i2.12671

Kumar, K., Singh D. R., Amaresan, N., \& Madhur, K. (2012). Isolation and pathogenicity of Colletotrichum spp. causing anthracnose of Indian mulberry (Morinda citrifolia) in tropical islands of Andaman and Nicobar, India. Phytoparasitica 40, 485-491.

León, J. \& Poveda, L. (2000). Nombres comunes de lãs plantas em Costa Rica. San José: Guayacán. 870 .

Lübeck, W. \& Hannes, H. (2001). Noni el valioso tesoro de los mares del sur. Madrid: EDAF. 173.

Mordue, J. E. M. (1971). Glomerella cingulata. In: Institute, C. M. (ed.) Kew Descriptions of Pathogenic Fungi and Bacteria.

Nelson, S. C. (2005). Species Profiles for Pacific Island Agroforestry. Recuperado de: http://nutriward.com/images/Morinda-noni.pdf. Acesso em: 29 jul. 2020 .

Poltronieri, L. S., Freire, F. C. O., Verzignassi, J. R., Ferreira, T. P. De S., \& Souza, A. C. (2009). Primeiro registro de Myrothecium roridum em noni no Brasil. Summa Phytopathologica, 35(1),69.

Potterat, O. \& Hamburger, M. (2007). Morinda citrifolia (Noni) Fruit- Phytochemistry, Pharmacology, Safety. Planta Médica. 73(3),1991-99. ISSN: 00320943.

Rao, U. S. M. \& Subramanian, S. M. (2009). Biochemical evaluation of antihyperglycemic and antioxidative effects of Morinda citrifolia fruit extract studied in streptozotocin-induced diabetic rats. Medicinal Chemistry Research. 18(6), 433-46. ISSN: 1554-8120.

Rethinam, P. \& Sivaraman, K. (2007). Noni (Morinda citrifolia L.) - the miracle fruit - a holistic review. International Journal of Noni Research, 2 (1-2), 4-37.

Silva, B. N. S., Ferreira, A. F. T. A. F., Mota, A. J., Bentes, J. L. S. (2019). Occurrence of noni anthracnose caused by Colletotrichum siamense in Amazonas, Brazil. Revista Agrária Acadêmica 2 (3). doi: 10.32406/v2n32019/7-9/agrariacad

Singh, D. (2012). Morinda citrifolia L. (Noni): A review of the scientific validation for its nutritional and therapeutic properties. Journal of Diabetes and Endocrinology, 3(6), 77-91.

Sousa, J. A., Aquino, A. R. L., Freire, F. C. O., \& Silva Neto, P. A. F. (2010). Produção de Mudas de Noni (Morinda citrifolia L.). Embrapa Agroindústria Tropical, Fortaleza, CE, Comunicado Técnico 157.

Sutton, B.C. (1992). The genus Glomerella and its anamorph. In: Colletotrichum: Biology, pathology and control. England, CAB International Wallingford, 126.

Wang, M. Y., Su, C. X., \& Chen, D. L. (2001). Cancer preventive effect of Morinda citrifolia (Noni). Annals of the New York Academy of Sciences. 952(1), 161-168. ISSN: 1749-6632.

Wang, M. Y., West, B., Jensen, C. J., Nowicki, D., Su, C., \& Palu, A. K., Anderson, G. (2002). Morinda citrifolia (Noni): A literature review and recent advances in Noni research. Acta Pharmacologica Sinica, 23, 1127-1141.

West, B. J., Ani, H., Palu, A. K., Tolson, C. B., Jensen, C. J. (2007). Safety tests and antinutrient analysis of noni (Morinda citrifolia L.) leaf. Wiley. Journal of the Science of Food and Agriculture. 87, 2583-88. 